\title{
L'ART DE DIRE ET DE FAIRE LE GOÛT : LES MÉDIATIONS CULTURELLES DANS UN MANGA CULINAIRE
}

\author{
Julie DERAMOND $^{1}$ et Emmanuelle LAMBERT ${ }^{2}$
}

\begin{abstract}
À travers l'étude monographique d'une bande dessinée japonaise (ou manga) spécialisée sur le vin et la cuisine (Les Gouttes de Dieu, de Tadashi Agi et Shu Okimoto), cet article analyse les questions de mise en correspondance des sens, de croisement des arts et d'interculturalité pour montrer comment s'élaborent des formes de médiation et de médiatisation des goûts culinaires, esthétiques et culturels. Le manga, considéré comme « médiaculture », se fait ainsi non seulement discours sur le vin mais également passeur de formes, d'objets et de valeurs culturels, devenant prescripteur. Entre discours expert et profane, terminologies professionnelle et autodidaxique, il s'apparente alors à une forme hybride de critique œnologique. Cette bande dessinée japonaise nous invite donc à penser les processus et discours communicationnels liés à la construction du goût, œnologique et esthétique.
\end{abstract}

1 Julie Deramond est maître de conférences en Sciences de l'Information et de la Communication et membre du Centre Norbert Elias, Avignon Université.

2 Emmanuelle Lambert est maître de conférences en Sciences de l'Information et de la Communication et membre du Laboratoire d'études et de recherches appliquées en sciences sociales, équipe MICS de l'Université de Toulouse-UPS.

Recherches en communication, n48 - Article publié le 26/02/2019 


\section{Introduction}

Qualifiée de «neuvième art » dans les années soixante, la bande dessinée a acquis depuis une reconnaissance croissante de la part des lecteurs et une légitimité de la part des chercheurs, en sémiologie, sciences de l'éducation comme en sciences de l'information et de la communication ${ }^{3}$. Parmi les industries créatives, elle constitue une forme de littérature populaire ainsi qu'un langage $^{4}$, en même temps qu'un phénomène de société, dont les codes sémiotiques sont déclinés dans de nombreux domaines d'expression à travers l'espace social, tels que le cinéma ou la publicité. Est-ce à dire que son pouvoir prescripteur peut contribuer à façonner les regards et les goûts ? Qu'en est-il plus particulièrement de l'influence de ce type de bande dessinée, le manga « gourmet» dit gurume manga, dans ce processus de construction ou de recomposition des représentations et des discours sur le vin?

L'objet de cette réflexion est celui d'un média culturel relevant des médiacultures, au sens de Maigret et Macé (2005). Nous analyserons ce manga dans ses dimensions anthropologique, esthétique, et médiationnelle, pour montrer comment se forme un discours sur le vin et à travers lui, comment s'élaborent de nouvelles formes de médiation et de médiatisation des goûts culinaires, esthétiques et culturels. Nous suivrons également les jalons posés par Antoine Hennion dans son manifeste pour une pragmatique du goût $(2004 ; 2005)$, et par Jean-Jacques Boutaud et al. autour d'une sémio-pragmatique du sensible (2003; 2010), en essayant de comprendre comment le goût se construit, se dit et ainsi, se partage.

Notre étude se fonde sur une analyse de contenu de la publication en série Les Gouttes de Dieu (Kami no Shizuku) de Tadashi $\mathrm{Agi}^{6}$ (textes) et Shu Okimoto (dessins), un manga pour adultes ou seinen, spécialisé dans le genre narratif de type « manga culi-

3 Voir Dacheux (2009); Escande-Gauquié \& Souchier (2011); Renard (2011).

4 Voir notamment Thierry Groensteen (1999) qui parle même de système.

5 La série se poursuit depuis 2016 en France avec Les Gouttes de Dieu - Mariage, chez Glénat (7 tomes parus).

6 Pseudonyme collectif de Shin Kibayashi et de sa sœur Yuko. 
naire » sur le vin ${ }^{7}$ paru au Japon entre 2004 et 2014 dans la revue Weekly Morning, publiée par Ködansha. Il obtient un succès mondial (près de 6 millions d'exemplaires vendus au Japon avant $2010^{8}, 3$ millions en Corée, et un million deux cent mille en France avant $2016^{\circ}$ ), entrant dans le cercle fermé des bestsellers. Nous étudierons les 31 premiers tomes ${ }^{10}$ de ce manga (sur les 44 que compte la collection chez Glénat), publiés en France entre novembre 2004 et janvier 2014. Pour aborder la médiation du goût par le biais d'un discours œnologique " grand public », la méthode sémio-pragmatique adoptée décrira l'univers visuel et sémantique du manga. Seront aussi mobilisés des commentaires de lecteurs sur des forums, quelques critiques de spécialistes et de bloggeurs et des articles de presse : qu'ils soient ainsi experts (sollicités dans les préfaces), ou lecteurs amateurs (laissant leurs avis via Internet), nous verrons comment leurs mots peuvent compléter nos analyses, et dans quelle mesure leurs discours peuvent devenir prescripteurs.

Comment un manga tel que Les Gouttes de Dieu, dit-il le goût du vin et peut-il participer à faire le goût en matière d'œnologie, alors même qu'il est écrit par des Japonais ? Comment cet aspect de la culture française, vu par le prisme d'un langage graphique populaire et extérieur à notre culture peut-il constituer un regard discursif singulier et prescripteur ? À travers cette analyse, nous montrerons successivement comment la mise en correspondance des sens, des arts et des cultures en un même discours, permet de tisser des médiations, pour mieux façonner le goût œnologique et esthétique.

7 Un autre manga seinen traduit en français traite du vin : la série Sommelier d'Araki Joh et Shinobu Kaitani (6 tomes, Glénat).

8 Marion Coquet, «Les Gouttes de Dieu, manga premier cru », Le Point, 17/10/2010.

9 Aurelia Vertaldi, «Les Gouttes de Dieu, à la recherche du vin divin », Le Figaro, 29/04/2016.

10 Nous avons cantonné notre étude aux 31 volumes sur les 44 , tout à fait représentatifs de l'intégralité de ce manga. 


\section{Au croisement des cultures, le goût}

Le goût, mais aussi le patrimoine culinaire et œnologique, sont des constructions sociales et font partie des « traits distinctifs » d'un patrimoine culturel immatériel (PCI) qui caractérise une société. Dans Les Gouttes de Dieu, le goût se construit en référence à une culture et par la rencontre avec d'autres cultures.

Le lecteur part en " quête du vin ${ }^{11}$, en suivant les traces du jeune Shizuku dans la tradition manga, puisqu' " "accomplir son rêve" " est le but ultime que tous les héros atteignent au prix d'affrontements répétés avec de "multiples adversaires" faisant du manga un produit culturel conçu pour mettre en scène "la volonté de puissance et le besoin d'accomplissement" " (Bouissou, 2006, $\S 30)$. Ce manga se fonde sur une construction assez manichéenne, axée autour de l'opposition entre cultures légitime et illégitime, savante et populaire, pour tenter de la dépasser. Ainsi, le choix d'une telle forme narrative n'a rien d'anodin : le manga est ainsi emblématique d'une culture populaire, souvent dépréciée en Occident (Bouissou, 2012, p. 19), même s'il fait l'objet depuis les années 2000 d'une réhabilitation certaine (Vanhée, 2012), en contradiction totale avec l'univers du vin - du moins des marchés historiques en France ${ }^{12}$-, qui se veut au contraire produit à fort capital symbolique, produit de consommation « culturelle » légitime et légitimé.

La trame du récit s'organise autour de la dispute de l'héritage d'un œnologue japonais disparu, Yutaka Kanzaki : Shizuku Kanzaki, le fils légitime, est totalement ignorant des techniques et terminologies liées à l'œnologie et à la viticulture (vignobles, crus, savoir-faire), mais il a une sensibilité et un palais hors du commun, ayant été initié au goût et à la bonne chère dès son enfance ; son rival, Issei Tomine, fils illégitime et adopté peu de temps avant la mort de Yutaka, est déjà un œnologue réputé internationalement, qui allie savoir encyclopédique et longue pratique de dégustation, même s'il n'a pas bénéficié de la même éducation au goût. Dans

11 T. 11, p. 219.

12 Certaines régions de production, voire une partie des vins dits du «nouveau monde » s'inscrivent aussi quant à eux dans un processus de recherche de légitimité. 
son testament, le père décrit douze grands vins, les douze apôtres, ainsi qu'un treizième mystérieux, vin «idéal » nommé Les Gouttes de Dieu. Chacun des fils devra retrouver quels vins se cachent précisément (cru, appellation, millésime) derrière les descriptions poétiques qu'il en a laissées. Les mots et les vins jalonnent ainsi leur apprentissage.

Afin de permettre au lecteur de s'identifier à ces héros, les auteurs insistent sur l'opposition classique entre expert et néophyte : le monde des initiés et de l'expérience affronte le monde du sensible et de l'émotion. Les termes utilisés s'inscrivent d'un côté dans des terminologies œnologiques techniques (de la décantation à la robe du vin, en passant par le millésime, le cru bourgeois ou le bouquet), de l'autre dans la description quasi intime des sensations et de l'imaginaire (avec la recrudescence d'expressions telle que « je ressens », « j'ai senti » ou « j'ai l'impression »...) : les personnages confrontent ou mêlent leurs points de vue, associant descriptions savantes et perceptions, mêlées d'impressions et de souvenirs. Le fil du récit est ainsi tissé autour de ces oppositions, pour se nourrir de références à l'univers du « roman initiatique » le plus classique, aux références artistiques largement partagées. Dès lors, c'est le choc des cultures que les auteurs mettent en scène, pour mieux tenter de l'atténuer dans la réalité : « Ce qui fait la force de ce manga $[. .$.$] c'est qu'il ouvre au lecteur un monde jusque-là$ réservé à un cercle d'initié $\rangle^{13}$. Ainsi les auteurs de manga créent des passerelles entre les genres et les goûts et détournent le genre littéraire du manga, en utilisant les ressorts d'un discours intermédiaire, qui ne tient ni véritablement du texte technique (Bray, 2010) ni purement du récit fictionnel, mais qui mêle tant dans le texte que dans le paratexte, fiction et factualité (Genette, 2004).

Des références constantes au réel permettent ainsi aux auteurs de créer des ponts entre cultures occidentale et asiatique : se rencontrent des goûts, des valeurs, des arts et des traditions différents. France et Japon sont au cœur de la mise en intrigue, même si des références à d'autres pays s'invitent au gré du récit, de l'Italie ${ }^{14}$ à

13 Jean-Loup, « un bon moment de lecture ! », 29/04/2012, Coin BD : http://www. coinbd.com/series-bd/les-gouttes-de-dieu/tome-1/

14 Meuble « Bussandri » dans le tome 13. 
la Corée ${ }^{15}$ en passant par l'Allemagne ${ }^{16}$ ou l'Autriche ${ }^{17}$ : le Japon parce qu'il est le berceau du manga et leur pays d'origine, se révèle par le biais de nombreuses allusions (paysages avec des vues sur Kobé, des jardins japonais d'autrefois ${ }^{18}$, Tokyo ou la montagne enneigée $\mathrm{d}^{\prime} \mathrm{Oze}^{19}$, mais aussi des références à $1^{\text {'artisanat }}{ }^{20}$, aux vêtements ${ }^{21}$ ou aux festivités traditionnelles ${ }^{22}$. L'autre pays qui colore le récit et les dessins reste la France, terre de vignerons, berceau du Champagne et du Saint-Émilion, sans doute en raison des représentations largement partagées au Japon qui font de la France un pays très populaire parce que berceau artistique et patrimonial et grand pays exportateur de vin. Tadashi Agi et Shu Okimoto distillent ainsi les allusions, reproduisant les cartes des grandes régions de production ${ }^{23}$, évoquant des vignerons méconnus mais bien réels (Boivin, 2011), alors que les grands crus français sont érigés en étalons de référence. Au-delà du vin, on notera de nombreuses références patrimoniales, qu'elles soient picturales, architecturales, touristiques (La Joconde, La tour Eiffel ${ }^{24}$ ), culturelles (Marché aux puces à Paris, carnaval25), ou commerciales (Vuitton, Chanel, Hermès $\left.{ }^{26}\right)$. Les archétypes, voire clichés touristiques, largement mobilisés sur le plan visuel pour évoquer la France ou d'autres pays européens, ne sont pas neutres ; ils peuvent incarner une image idéalisée des auteurs japonais, ou s'apparenter à une démarche de marketing culturel.

L'interculturalité est ainsi tant intradiégétique, relative aux nombreuses relations qu'entretiennent les personnages avec

15 Du Kimchi au Bibimbap dans les tomes 12 et 13, par exemple.

16 Château de Neuschwanstein dans le tome 6.

17 Avec le Baiser de Klimt, dans le tome 22.

18 T. 10, p. 201.

19 T. 17 , p. 16-17.

20 Les Karuizawa Bori, des meubles en bois, dans le T. 15, p. 202.

21 Le Yukata, kimono d'été, T. 12, p. 93.

22 La fête des pêchers, le Momo No Sekku, T. 24, p. 48.

23 Par exemple dans le T. 17, la carte de la Bourgogne et les grandes régions de production du vin blanc.

24 T. 7, p. 181.

25 T. 4, p. 22 et p. 61.

26 T. 6 , p. 103. 
d'autres cultures, en circulant du Japon vers la France, la Corée, la Suisse ou les États-Unis, qu'extradiégétique, puisque la série emprunte - dans une large intertextualité - tant aux codes de la littérature japonaise (manga), qu'aux codes culturels français. Pour Jean-Luc Poignard, «Le double exploit d'Agi et Okimoto se révèle dans l'ouverture de deux mondes l'un à l'autre. Nous pénétrons la sensibilité japonaise et leur culture du vin, tandis qu'ils se livrent à un plaidoyer de nos flacons les plus réputés $»^{27}$. De nombreux exemples illustrent ces références aux codes culturels des deux mondes, telle cette allusion à l'étiquette d'un château Calon Ségur St Estèphe, 2000, en forme de cœur, ce qui « en fait un vin très recherché pour la St Valentin », suivant la tradition japonaise ${ }^{28}$. Les codes culturels s'expriment aussi par l'expression des habitus, dans les « accords mets et vins », ou les codes de proxémie par exemple. Ainsi, le « bon goût » relève d'un mélange assumé des cultures : regard sur un paysage japonais ${ }^{29}$ par des œuvres d'art occidentales, accord entre les saveurs de Corée et les vins d'un terroir italien ${ }^{30}$.

Plus largement, les historiettes qui parsèment le récit mettent en exergue la mixité heureuse des cultures, en « parfaite harmonie ». Le tome 14 démontre avec originalité qu'une culture « nationale » tient du regard que l'on porte sur elle : un des personnages doit concocter un plat qui puisse être typique à la fois pour un Français et un Japonais ${ }^{31}$. Un plat à base de thon sera ainsi décrit par le Japonais, comme « un sashimi de thon rouge » et un « tataki de thon enveloppé dans du Nikogori » ${ }^{32}$, quand le Français y verra un « carpaccio de thon recouvert de sa sauce aromatique à base d'huile de sésame » avec un thon « tranché et accommodé en aspic $\|^{33}$. Le même plat renvoie à des termes culinaires spéci-

27 Jean-Luc Poignard, « Un manga dans le monde du vin », Génération Vigneron, 27/07/2017 : http:www.generationvignerons.com

28 Voir la note du traducteur, explicite. T. 2, p. 68.

29 T. 14, p. 103 : Le pont de Waterloo d'André Derain pour la vue de Tokyo.

30 Pour le kimchi, plat coréen pimenté et le Gravello, vin du sud de la Calabre (T. 13).

31 T. 14, p. 115.

32 T. 14, p. 159.

33 T. 14 , p. 161. 
fiques, et à des traditions nationales anciennes ; il s'agit de démontrer qu'au-delà des cultures, le bon goût se partage aisément. Le manga participe aussi d'une envie de rencontrer l'autre à travers son alimentation et sa cuisine, comme en témoigne Jean-Nicolas Méo, directeur du domaine de Méo-Camuzet (Bourgogne) : «Les Japonais viennent dans nos propriétés pour goûter nos vins. Certains apprennent même le français pour l'occasion $»^{34}$.

\section{Correspondances des sens}

Les mangas atteignent une consécration culturelle en France dans les années 2000, notamment ceux dits d'auteur, car ils réussissent à réunir des éléments diégétiques liés au quotidien, à l'introspection, ou à la contemplation et « à figurer les réalités immatérielles de "l'intériorité" [ce qui] contribue ainsi à asseoir leur légitimité » (Vanhée, 2012, p. 159). Les Gouttes de Dieu parvient à traduire graphiquement les émotions, les sensations éprouvées à la dégustation : tout ce qui est difficile à exprimer sans le recours à la fiction et à l'image. Ainsi, analyser ce manga par le prisme des correspondances de sens permet de sonder les mystères de l'expérience de la dégustation, et voir comment les auteurs retranscrivent l'ineffable des sensations, en traduisant « l'espace figuratif du culinaire » (Boutaud \& Madelon, 2010), par le biais de mots, d'images et de références tant artistiques que sensorielles.

L'apprentissage se nourrit du souvenir et de la mémoire. Les auteurs entrelacent ainsi le récit de références au passé, en convoquant des historiettes liées à la filiation et à l'héritage. C'est le premier levier des descriptions de vins qui jalonnent le parcours initiatique. Shizuku décrit même un vin en référence à la figure paternelle : " exprimer ce vin d'un mot... mais si j'osais quand même... [c']est mon père $\aleph^{35}$. Le rapport au temps s'exprime également par des descriptions fines de paysages et d'instants vécus, dans des registres poétiques et imagés emprunts de nostalgie et de tendresse : «c'est un crépuscule d'été à l'approche de l'automne »,

34 Cité par Sébastien Julian, «Les Gouttes de Dieu, le manga japonais dont nos vins sont les héros », L'Express, 24/09/2010.

35 Les Gouttes de Dieu, T. 30, p. 103. 
« en inhalant ses arômes, j'ai l'impression de flotter, comme si j'étais emmené sur une plaine de montagne... » ${ }^{36}$. Le passé prend corps dans les monuments qui parsèment les dessins, se confrontant à l'architecture du présent et au monde d'aujourd'hui ${ }^{37}$. Ainsi l'importance de la transmission du goût, de père en fils est-elle matérialisée au fil du récit pour mieux inscrire le vin dans le processus de patrimonialisation, véritable héritage légué du passé (Davallon, 2006).

Sur le plan stylistique, le discours mobilise essentiellement le croisement des arts dans les descriptions. Celles de dégustation du vin convoquent une intertextualité liée à une interdisciplinarité revendiquée : outre les pays et paysages visités, sont décrites et illustrées des œuvres d'art (tableaux pour la majorité, sculptures, monuments, œuvres musicales ou chorégraphiques), comme les pratiques et expressions du patrimoine immatériel (traditions, art de vivre ou sport). Ces références apparaissent sur un plan métaphorique au moment de la dégustation, afin de rendre plus expressif le ressenti : référence au cinéma pour décrire un Clos-Vougeot 1996, un vin « riche, dense [...] ça me fait penser à "Vacances romaines" le célèbre film d'Audrey Hepburn ${ }^{38}$; aux arts décoratifs avec une bouteille de Champagne « dessinée par le maître verrier Émile Gallé représentant du style art nouveau ${ }^{39}$; ou encore à la danse classique La Bayadère se mêlant à la danse indienne antique ${ }^{40}$. Souvent, les œuvres convoquées dans la description des vins sont des chefs-d'œuvre ou des symboles culturels emblématiques : la statue du David de Michel-Ange à Florence « se tenant debout dans une pose à la fois virile et gracieuse $\gg^{41}$, la Victoire de Samothrace, ou La dentellière de Vermeer. Ingres, Degas, Delacroix, Van Gogh, Monet, Derain, sont les représentants du patrimoine pictural français, de même que Jean-François Millet, avec $L$ ' $A n$ -

36 T. 27 , respectivement p. 63 et p. 165.

37 T. 27, respectivement p. 146 et p. 148.

38 Sic. Film de William Wyler (1953), avec Audrey Hepburn dans le rôle principal.

T. 12, p. 152-153.

39 T. 15, p. 178.

40 T. 16 , p. $60-61$.

41 T. 26, p. 131. 
gélus, « ce fameux tableau qui décrit la silencieuse prière pour les bienfaits de la terre... ce vin exprime un appel au terroir $[\ldots]$ j'ai l'impression de me retrouver au cœur de ce chef-d'œuvre $»^{42}$. Ces références constituent des stéréotypes « économe(s), stable(s), consensuel(les) » (Boyer, 2008), autrement dit, immédiats et rassurants. À travers ces déclinaisons métaphoriques s'observe une vision assez stéréotypée de l'identité et de la culture occidentales, réduites parfois à des images d'Épinal ou à des icônes, telle $L a$ Joconde, dont l'image quasi subliminale traverse les tomes (avec des variations en fonction des vins qui l'évoquent, elle apparaît même sous les traits d'une femme enceinte, évocation de la mère) $)^{43}$ et résume à elle seule l'ambition des auteurs, toujours attentifs à « mettre en culture » le vin en suivant la dynamique de l' « assomption ${ }^{44}$, d'après la définition d'Emmanuel Souchier et Yves Jeanneret (1999, p. 74). Pour autant, il s'agit toujours de mettre en correspondance ces vins avec des valeurs largement partagées : valeur patrimoniale, mais aussi technique, historique, esthétique, économique... liée à des savoir-faire, des formes culturelles ou sacrées.

À travers cette multiplicité d'expressions artistiques, s'incarnent également des correspondances de sens en une « rhétorique évocatrice » (Souchier \& Jeanneret, 1999, p. 72) : tous les sens sont mis en émoi et sollicités, avec une primauté évidente accordée à la vue sur ce support graphique. La gageure pour les deux héros est de pouvoir exprimer précisément leur ressenti par des images, des métaphores : trouver les accords justes ou mettre en correspondance le goût avec la vue, l'odorat, le toucher ou l'ouïe. Les images suggèrent des stimuli ou des sensations liés aux autres sens : ainsi se multiplient les « signes sensoriels, à caractère indiciel par rapport aux sensations gustatives : le plus souvent par synesthésie de formes, de couleurs, de proportions » (Boutaud \& Madelon, 2010, p. 35) afin de rappeler au gré des pages que

42 T. 2 , p. 102 et T. 1, p. $88-89$.

43 T. 10 , p. 12 ; T. 16 ; T. 18 , p. 129 ; T. 28 , p. 198.

44 «L'assomption réside dans le fait d'associer le vin à des motifs chargés d'une valeur reconnue dans la société ». 
« le vin $[\ldots]$ n'est pas un simple alcool. C'est un livre, c'est un tableau... $»^{45}$.

La synesthésie décloisonne les codes de la dégustation centrée sur un discours expert pour s'ouvrir au langage du corps. Elle stimule l'imagination par un kaléidoscope de possibilités et d'hybridations, et si les cinq sens sont sollicités, certaines stimulations sensorielles sont plus insolites, tel ce mélange des genres entre goût et son, dans le tome 1 pour évoquer un Château Mont-Pérat de 2001 : « Il est puissant avec un goût sucré et fondant où soudain survient une note d'acidité ! C'est comme la voix suave de Freddie Mercury enveloppée par les riffs de guitare et le bruit sourd de la batterie... Comment dire... ça ressemble à du classique mais ça n'en est pas ${ }^{46}$. Côté visuel, le Charmes Chambertin 2003 de Philippe Pacalet est comparé au tableau d'Hokusai, La grande vague de Kanagawa. Plus originale est la comparaison d'un vin, dans le tome 6, avec un temple oriental dans lequel flotte " l'odeur discrète de l'encens $\gg{ }^{47}$. Goût et odeur sont souvent proches (Candau, 2005 ; Pellitteri, 2007) et entrent moins bien en résonance avec le visuel et le son, sans doute plus adaptés pour dire le goût.

Au-delà même des sens, le corps semble partout présent dans le manga afin de rappeler au lecteur la nécessaire implication de tout son être, lors de la dégustation, pour une véritable incorporation : sensualité ${ }^{48}$, féminité, puissance, mais aussi sueurs et larmes sont autant d'éléments convoqués pour compléter cette approche sensible, voire charnelle, du vin. L'émotion peut alors être invoquée par les auteurs, afin de faire ressentir que la dégustation tient de l'expérience : l'émotion est mise en scène par les mots et par l'image, évoquée au travers de nombreuses scènes largement illustrées, de l'attendrissement, à la liesse populaire du carnaval ${ }^{49}$, en passant par la catastrophe $\mathrm{e}^{50}$, la violence du spectacle taurin ${ }^{51}$ ou la

45 T. 4, p. 161.

46 T. 1, p. 109.

47 T. 6, p. 140.

48 T. 1, p. $182-183$.

49 T. 4, p. 61-66.

50 Tsunami dans la région de Sendai, en 2011 : T. 31.

51 T. 13, p. 124. 
joie enfantine ${ }^{52}$. Et les auteurs utilisent toute la palette propre à la forme littéraire graphique du manga, suivant les codes et canons du genre, pour mettre en valeur l'effet du vin sur les corps et les ressentis des personnages, qui transparaissent jusque sur leur visage $^{53}$. Un tel parti pris en vient même à agacer certains lecteurs, comme l'atteste ce commentaire : " cette manie $[\ldots]$ d'en faire des tonnes dans les émotions me gave sévère [...] mais c'est incroyaaaable ! Ohlalalalala, je n'avais jamais rien goûté de tel avant ! AAAaaaAAAaaah ! ${ }^{54}$. D'un point de vue visuel, l'emphase et l'exagération dominent aussi, mais alternent selon les situations et les émotions décrites, avec un dessin plus sobre et épuré ; le rythme des séquences de cadres est quant à lui assez dynamique et alterne régulièrement avec des doubles pages où se déploient les descriptions métaphoriques et graphiques hyperréalistes très détaillées. En témoigne la double page du T. 4 présentant Yutaka Kanzaki en pleine dégustation : le verre de vin étant mis en relation avec des tableaux d'une grande précision. On notera par ailleurs l'alternance des plans pour montrer le verre de vin, en gros plan en pied, posé sur une table, puis tenu à la main, au milieu de l'image, comme grossi par rapport à la taille du personnage, puis en plongée, pour mieux mettre en valeur le ressenti de Yutaka lorsqu'il hume le vin avant de le boire.

Cette série traduit bien l'idée que la transmission des connaissances sur le vin et l'art de la dégustation, qu'il s'agisse de technique, de savoir-faire, comme de terminologie, peut se faire sur un mode gourmand incarné, par un apprentissage sensoriel auquel les correspondances culinaires et artistiques peuvent contribuer. En creux, ce manga questionne les différentes façons dont les saveurs et leur description peuvent servir la médiation (Lambert, 2009), et au-delà d'une initiation (aux goûts), comment elles peuvent inviter à l'appréciation esthétique. Le choix des vins et autres " ingrédients » culinaires dégustés, et leur mise en relation avec des œuvres d'art, tout cela participe à la construction du/des sens,

52 T. 2 , p. 195.

53 T. 14, p. 156.

54 Le moine (37 ans), 14/03/2009. BD Gest', consulté le 20/01/2015 : http://www.bdgest.com/forum/les-gouttes-de-dieu-t11022-20.html 
selon une approche sémio-pragmatique du sensible (Boutaud \& Lardellier, 2003). Ainsi, à l'image du vin que l'on élève, les correspondances de sens et les jeux de synesthésie créent une stylisation, voire une spectacularisation des représentations du goût, tout en construisant des médiations et en contribuant à une éducation des goûts esthétiques et culturels.

\section{Construire le goût}

Le goût se fait en se disant et se dit en se faisant. L'outil de la réflexivité tend à prendre la forme plus classique de l'écrit et de façon très caractéristique, chaque domaine donne naissance à un vocabulaire spécifique, plus ou moins développé qui vient se loger entre la description physiologique ou technique des objets et le rendu littéraire des émois de l'amateur. À travers ces expressions, par exemple sur le goût du vin (les fruits rouges, les racines, les champignons, la truffe, le boisé...) ni purement techniques, ni seulement imagées, le goût se repère, s'instrumente et peut se partager avec d'autres. (Hennion, 2005, p. 8).

Ainsi, le manga tente d'exprimer, en mêlant images et symboles culturels, "ce qui se passe " (ibid.) lorsqu'un amateur déguste un vin. C'est justement ce partage, qui s'établit par des mots, des images, et des références collectives, qui contribue à aider les lecteurs à élaborer leur propre goût, leur propre jugement. Il est d'autant plus important de proposer de telles images que le « langage naturel des odeurs se caractérise par son imprécision et son instabilité » (Candau, 2005). Et si l'on ne maîtrise pas la terminologie technique, il faut alors passer par une autre rhétorique, plus poétique : « la perspicacité des papilles et la finesse de l'odorat se forment sans nul doute dans un dialogue assidu avec les bonnes bouteilles, mais il faut aussi apprendre le vin dans les livres » (Albert, 1989) et apprendre ainsi à maîtriser la grammaire du vin.

Les Gouttes de Dieu est conçu comme un pont entre l'univers livresque et encyclopédique des connaisseurs et le ressenti instinctif des néophytes, les initiant progressivement dans leur 
« carrière » d'amateur, selon Antoine Hennion (2005). Ce dernier évoque quatre éléments prédominants, piliers de la construction du goût de tout amateur, qui sont chacun présents dans ce récit : « l'objet goûté, le collectif des amateurs, les dispositifs et conditions de la dégustation, le corps qui ressent» (Hennion, 2005, p. 8). Le véritable héros du manga est bien évidemment le vin qui est au cœur de tous les épisodes, à commencer par l'étiquette dessinée avec une précision photographique, ce qui symbolise, par métonymie, l'extrême soin qui est accordé à la description du vin : « l'étiquette n'est pas seulement étiquetage ; elle est pièce d'un scénario de dévoilement graduel du corps du vin [...] elle annonce temporellement la promesse de son excellence » (Souchier \& Jeanneret, 1999 , p. 74). On peut observer cela dans le tome $5:$ le visage très expressif de Miyabi semble s'effacer devant les deux bouteilles de Bonnes Mares dessinées en gros plan avec le plus grand détail, et comme auréolées d'un éclat lumineux à l'arrière-plan, pour mieux souligner leur qualité aux yeux du lecteur.

D'autres « sémiotiques-objets » (Fontanille, 2005) accompagnent l'étiquette pour mieux décrire « l'objet goûté » en créant un véritable « univers sémiotique » lié à l'œnologie : le verre, la bouteille, le bouchon, la carafe, le bar à vin... sans oublier les techniques et savoir-faire liés à la viticulture : le terroir, le château ou le domaine, le travail du vigneron, les vendanges... Les mangakas apportent également un soin particulier à la description des conditions de dégustation, scénarisée méticuleusement : le vin n'est pas apprécié de la même manière selon qu'il est bu seul, dans son salon, chez un caviste, en plein désert ou dans une situation conviviale, avec des amis, de simples connaissances, à l'apéritif ou devant un plat de choix, etc. Les dispositifs liés à la dégustation sont aussi révélateurs : le geste de la décantation du vin ${ }^{55}$, pour initier le lecteur à l'un des rituels les plus symboliques, distingue largement selon son degré de maîtrise l'amateur du profane. On ne reviendra pas sur l'importance du « corps qui ressent » (Hennion, 2005, p. 8), matérialisée par de longues séquences de dégustation, mettant en scène les personnages qui expriment leur perception

55 Par exemple, T. 15, p. 83. 
du vin, et les images puissantes qu'un vin peut éveiller. Mais l'on soulignera ici davantage la nécessité de mobiliser le « collectif des amateurs » dans cette quête du goût : « il faut se mettre ensemble (ce peut être la réunion physique, comme c'est souvent le cas, mais cela peut être simplement l'appui indirect sur une communauté, sur des traditions, sur des récits et des écrits, ou sur le goût des autres) » (Hennion, 2005, p. 8). Le tome 13 s'achève ainsi sur Shizuku en gros plan, concentré dans sa dégustation, puis sur les amis qu'il a rencontré durant l'épisode. La morale de l'histoire évoque la nécessité de trinquer ensemble, devant un bon verre de vin; elle est matérialisée dans l'avant-dernière case par un personnage portant un toast verre levé, et dans la dernière, par le groupe d'amis réuni autour du vin et du terme fédérateur et évocateur entre tous en français : « Santé !»

Au fil des pages, les auteurs ne cessent ainsi de montrer à quel point la perception se nourrit de celle des autres : le héros Shizuku avance dans son initiation grâce aux nombreux personnages qui l'entourent et le guident, distillant au gré des bulles, connaissances livresques, descriptions savantes, souvenirs emprunts de nostalgie ou émulation féconde, selon qu'ils sont amis (Miyabi), maîtres ou figures paternelles (Robert Doi, M. Fujeida), ou rivaux (Issei Tomine). Si la communauté (familiale, amicale) est un pilier de la fiction, elle semble devenir une réalité pour les lecteurs les plus assidus, qui échangent sur Internet leurs points de vue sur ce manga. Des forums sur des sites dédiés à la BD ou au vin ( $L a$ Revue du Vin de France, Coin BD, BD Gest' $)^{56}$ rassemblent des commentaires de fans de mangas ou de lecteurs curieux, d'abord amateurs de vin, les aidant ainsi à construire leur propre carrière d'« amateur » en partageant avec d'autres leurs expériences.

56 Messages publiés (2010-2012) sur La Revue du vin de France : http://forum.larvf. com/larevuedesvinsdefrance/carte-blanche/gouttes-dieu-sujet_29_1.htm ; 113 messages recueillis (2008-2014) sur BD Gest' : https://www.bdgest.com/forum/lesgouttes-de-dieu-t11022-100.html; entre 2 et 6 avis par tome, sur Coin BD. Pour ce lien : les avis sont maintenant affichés à la fin de chaque page dédiée à un tome précisément. Par exemple : http://www.coinbd.com/series-bd/les-gouttes-de-dieu/tome-4/ 


\section{Le pouvoir prescripteur du manga}

L'analyse des commentaires publiés (2008-2010) liés à la lecture des Gouttes de Dieu, permet d'observer à quel point ce manga suscite la curiosité pour le vin et l'envie d'en faire l'expérience ${ }^{57}$, à l'image de Gala 35 qui écrit qu'il lui «a donné envie de goûter à du Bourgogne $»^{58}$ alors que Banana Joe constate : "plus je lis et plus j'ai soif, ça c'est plaisant $»^{59}$. La lecture conduit vers l'initiation : Yaneck explique ainsi avoir voulu, à « la façon dont ils parlaient du vin dans le manga, [...] faire pareil » alors qu'il «n'aimai(t) pas le vin $»^{60}$. Il se déplace alors pour « une séance d'œnologie chez un caviste grenoblois $»^{61}$. Plus qu'à goûter le vin, les lecteurs sont invités à devenir de véritables amateurs, en se «plonge(ant) [...] dans le monde du vin $»^{62}$.

Au fil du récit, l'itinéraire de Shizuku enseigne au lecteur que l'amour, voire la passion du vin (Demossier, 2002), doit satisfaire à certaines exigences : déguster beaucoup ${ }^{63}$, connaître son histoire et ses techniques de fabrication, connaître les gestes ${ }^{64}$ et le vocabulaire afférents, mais aussi s'impliquer personnellement (l'associer à ses souvenirs ${ }^{65}$ voire risquer de sacrifier sa vie pour lui $\left.{ }^{66}\right)$. Les Gouttes de Dieu contribue alors à façonner le goût de ses lecteurs, par la mise en récit et en images de l'apprentissage et de l'expérience de dégustation, autant que par des pages plus encyclopédiques publiées en fin d'ouvrage : « le B.a-ba de l'œnologie » et

57 L'illustration par les commentaires de lecteurs vient en contrepoint de nos observations, elle ne vise pas la représentativité ; et notre étude n'étant pas centrée sur la réception du manga, elle ne permet pas ici de dresser de typologie des lecteurs et/ou amateurs de vin, ni de montrer les corrélations entre la lecture de la BD et l'achat de vin.

58 Gala 35 (35 ans, habitant à Rennes), 12/04/2008. BD Gest', ibid.

59 Banana Joe, 28/07/2010, ibid.

60 Yaneck (32 ans), Sévigné, 15/02/2010, ibid.

61 Yaneck, ibid.

62 «Moi » (39 ans), Saint-Amand-Les-Eaux, 11/11/2008, ibid.

63 T. 24, p. 41 : «Il n’y a pas d'autre méthode pour connaître le vin que de le goûter. » 64 Voir Stengel (2017).

65 T. 32, en Bourgogne.

66 T. 17, p. 163. 
« Enologie pratique », où « deux experts vous invitent dans le monde fascinant du vin!».

Loin de l'image stéréotypique d'une littérature de divertissement, ce manga utilise les ressorts narratifs pour amener les lecteurs à apprendre le vocabulaire œnologique, et par là entrer dans le monde expert du vin. Aux versants ludique et fictionnel s'adjoint la dimension didactique par les nombreux éléments d'explication tant sur la viticulture que sur l'œnotourisme, en utilisant les termes consacrés : des cépages aux appellations, en passant par les techniques de vinification ${ }^{67}$ ou de dégustation. Le manga est jugé très « instructif $»^{68}$ et 《 didactique $»^{69}$ par les lecteurs car il crée de bonnes conditions pour les inciter à s'informer et à approfondir leurs connaissances. Ainsi, un lecteur dont « la cave, plus que (la) bibliothèque est celle d'un "amateur éclairé" ", s'avère " plutôt bluffé » « par la recherche des auteurs, tout d'abord » qui évoquent de « vraies » appellations et donnent un « cours d'œnologie » de manière "didactique et accessible ${ }^{70}$. Impression confirmée par l'œnologue Michel Dovaz qui écrit que ce manga « désacralise le vin, trop souvent traité avec technicité et tristesse $»^{71}$ et s'apparente à un " manuel du vin français pour les nuls $\|^{72}$. Entre érudition et vulgarisation, " c'est donc aussi à un apprentissage à contre-courant, guidé par la curiosité et l'ouverture d'esprit que nous convient les auteurs $>{ }^{73}$, participant à la transmission, voire à la construction de savoirs.

La portée de cet ouvrage ne se limite pas à ce champ des savoirs. Nombre de lecteurs partagent leur expérience personnelle, décrivant leurs propres souvenirs de dégustation. Banana Joe écrit :

67 Par exemple les termes utilisés : arôme, bouchonné, décantation, dégustation à l'aveugle, domaine, lie, etc., sont définis dans le « vocabulaire pour débutants », T. 2, p. 226-227.

68 Horace G. (59 ans), 26/10/2009. BD Gest', ibid.

69 « Des interviews et (d)es rajouts en fin de livre... très intéressants » : Alley Opp, 8/10/2008, ibid.

70 Eyesparkle, 17/08/2011, ibid.

71 Michel Dovaz, « Préface », T. 1.

72 Gaël Vaillant, « Un manga dope le vin français », Le JDD, 24/09/2010 : https://www. lejdd.fr/Style-de-vie/Vin/Un-manga-dope-le-vin-francais-222518-3254030

73 Eyesparkle, 17/08/2011, BD Gest', ibid. 
« stupeur, je me suis plongé dans cet ouvrage me rappelant certaines senteurs, certaines sensations déjà vécues. Je me souviens de mon premier Sauternes 1978, je l'avais qualifié de "pipi de bon Dieu" $"{ }^{74}$. Les bulles et planches graphiques du manga réactivent d'après les lecteurs, des souvenirs oubliés ou stimulent leur imagination, même si les commentateurs restent critiques devant l'exagération des traits et des situations : « il m'est arrivé à mon modeste niveau de me prendre à rêver à des paysages en buvant une bonne bouteille ou à me poser la question : si ce vin était de la musique, laquelle serait-ce ? (de là à entendre Freddie Mercury, il y a une marge) $\rangle^{75}$. Ainsi, le manga semble réussir à transformer, chez certains lecteurs, leur propre expérience de dégustation.

Les articles de presse expliquent à quel point ce manga entraîne ses lecteurs vers la quête du vin, mettant en exergue son pouvoir prescripteur, à l'instar d'autres médias plus collaboratifs sur le web (Lambert \& Trouche, 2008). En mêlant fiction et réel et en donnant des détails précis sur de vrais vignerons et des crus commercialisés, Les Gouttes de Dieu se transforme ainsi en véritable « guide » œnologique, tels les célèbres guides Hachette et Parker. Les importateurs ${ }^{76}$ comme les particuliers suivent alors les recommandations des auteurs pour acheter leurs vins en Europe, comme en témoignent Zanzibar qui « note les noms des vins et des années $»{ }^{77}$ cités afin de les acheter, ou Banana Joe qui « avale les tomes 6 à 12 de la série », mais déplore de ne « pouvoir goûter tous ces vins décrits », car ils ne sont pas à sa portée ou « tout simplement plus dispo[nibles] $»^{78}$. Enfin, l'« effet manga » ainsi que la publicité des vins, ne tiennent pas de la fiction : les retombées financières qu'il fait dégager aux vignerons cités ne sont guère négligeables. Philippe Carille dont le Château Poupille 1999 apparaît dans le tome 9, aurait pu « vendre quinze fois (s)a récolte »,

74 Banana Joe, 26/10/2009, ibid.

75 Horace G. (59 ans), 29/09/2010 ibid.

76 Voir Marie-Anne Lubin, «Les Gouttes de Dieu : Ce vin est la passion cachée », La Revue du vin de France, 13/11/2013.

77 Zanzibar, 3/02/2010, BD Gest', ibid.

78 Banana Joe, 28/07/2010, ibid. 
son volume d'activité ayant augmenté de $30 \%$, selon l'Express ${ }^{79}$. Au point que Thibault Despagne, producteur du Mont-Pérat considère le manga, comme un « levier exceptionnel, bien plus efficace que les points Parker $»^{80}$.

Ainsi, le vin se transforme en produit culturel et le média en guide critique $^{81}$, mis en abyme à l'intérieur du récit de fiction. Ce sont alors l'indépendance et le désintéressement des auteurs qui garantissent l'authenticité du jugement critique (Béra, 2003). Les différentes énonciations constituent à proprement parler des médiations, du moins si l'on considère l'action du médiateur dans sa fonction de pédagogue, ou de « passeur » (Caillet, 1995), c'està-dire « celui qui accompagne, plus que celui qui distribue du savoir tout mâché qui consiste à permettre à un sujet social de produire, à partir d'une expérience inédite à laquelle le médiateur le confronte, les connaissances nécessaires à l'accès à d'autres formes de représentation de la réalité concrète » (Péquignot, 2007, p. 7). Si ce manga relève donc d'une dimension critique, dans les appréciations portées sur le vin ou les œuvres d'art, c'est dans le sens d'une énonciation contribuant à « penser l'œuvre et à penser à partir de l'œuvre elle-même » (Kantcheff, 2005), et la fabrique du goût, en tant que médiation, ne vise ni injonction savante, ni jugement péremptoire : elle déploie des possibles.

\section{Conclusion}

Au-delà des représentations et réceptions convenues, Les Gouttes de Dieu semble initier le lecteur au goût du vin et à l'expérience de la dégustation. Par le prisme du croisement des arts (peinture, sculpture, architecture notamment), et des accords mets et vins (rapprochant cuisine asiatique et européenne de crus euro-

79 Sébastien Julian, «Les Gouttes de Dieu, le manga japonais dont nos vins sont les héros », op. cit. ; voir également Anne-Laure Pham et Sébastien Julian, « Un manga fait s'envoler le prix du vin », L'Express Tendances, 24/09/2010.

80 Cité par Sébastien Julian, L'Express, op. cit.

81 La reconnaissance du manga se traduit par les récompenses obtenues : « meilleur livre du monde sur le vin » lors du Gourmand World Coobook Awards (2009), Meilleur Seinen à Polymanga (2009) et aux Japan Expo Awards (2011) et Grand prix de La Revue du vin de France en 2010. 
péens, australiens ou américains), la médiation du vin devient une invitation au voyage, qui transcende les cultures. Cette valorisation du patrimoine vinicole et culinaire est d'autant plus riche qu'elle s'opère à travers un regard étranger, celui des auteurs japonais, et un discours de fiction adressé à des profanes.

Quand la découverte œnologique mène à la rencontre de l'autre, et la médiation du vin s'accorde à la médiation interculturelle, le manga se fait alors non seulement passeur de formes, d'objets et de valeurs culturels, mais il devient aussi prescripteur de goût (de goûts éclectiques), s'apparentant à une forme hybride de critique œnologique. Par ce processus de circulation et de réappropriation de codes visuels, de références culturelles, et de discours professionnels, Les Gouttes de Dieu incarne une forme de diffusion et de vulgarisation, voire de trivialité ou de trivialisation (Jeanneret, 2008) qui met en scène la circulation des êtres culturels dans la vie sociale, en mobilisant ici synesthésie, interculturalité et médiations. Le jugement d'un expert, Philippe Bourguignon, meilleur sommelier de France en 1978, résume bien les ingrédients de cette série : « peut-être que la solution est là, dans cette lecture "manga" autour du vin où le regard extérieur sur notre métier offre mille facettes de l'apprentissage ; tout ce qui nous semble évident, ordinaire, naturel, familier, presque inné est ici décortiqué, amplifié, mis en scène. [...] Fascinante lecture en définitive qui, sans se prendre au sérieux, initie au monde du vin souvent trop hermétique. L'essentiel étant de mener à une meilleure compréhension du vin par une connaissance joyeuse et non hautaine et au partage de la table et à l'ivresse des mots qu'elle engendre ${ }^{82}$. Entre discours expert et profane, terminologies professionnelle et autodidaxique, quand la dégustation se mêle à l'éloquence, ce manga maîtrise l'art de dire et de faire le goût des autres, des amateurs qui apprennent à aimer.

82 T. 2 , p. 6. 


\section{Références}

Albert, J. P. (1989). La nouvelle culture du vin. Terrain 13, 117-124.

Béra, M. (2003). Critique d'art et/ou promotion culturelle ? Hermès 117, 153-187.

Boivin, N. (2011). De la géographique à travers un manga « viticole »: l'exemple des Gouttes de Dieu. Bulletin de l'association des Géographes français, 67-81.

Bouissou, J. M. (2012). Manga : Histoire et univers de la bande dessinée japonaise. Arles : Picquier Poche.

Bouissou, J. M. (2006). Pourquoi aimons-nous le manga : Une approche économique du nouveau soft power japonais. Cités, 27. Disponible à : https://www.cairn.info/ revue-cites-2006-3-page-71.htm.

Boutaud, J. J. \& Lardellier, P. (2003). Sémio-anthropologie du sensible. Degrés 113.

Boutaud, J. J. \& Madelon, V. (2010). La médiatisation du culinaire. Communication \& Langages 164.

Boyer, H. (2008). Stéréotype, emblème, mythe. Sémiotisation médiatique et figement représentationnel. Mots, les langages du politique 88, 99-113.

Bray, F. (2010). Qu'est-ce qu'un texte technique ? Réflexions à propos de la Chine et des Andes. Techniques \& Culture 54-55, 185-199. Disponible à : http://journals. openedition.org/tc/4997. Doi: 10.4000/tc.4997.

Caillet, E. (1995). À l'approche du musée, la médiation culturelle. Coll. Muséologies. Lyon : PUL.

Candau, J. (2005). Vins, arômes, couleurs et descripteurs sensoriels. Quel partage de la dégustation? MEI, Le corps, le vin et les images 23, 23-38.

Dacheux, E. (2009). La bande dessinée, art reconnu, média méconnu. Hermès 54.

Davallon, J. (2006). Le don du patrimoine : une approche communicationnelle de la patrimonialisation. Paris : Hermès Science - Lavoisier.

Demossier, M. (2002). Les passionnés du vin ou le mariage du cœur et de la raison. Dans C. Bomberger (dir.). Passions ordinaires. Paris : Hachette, 163-188.

Escande-Gauquié, P. \& Souchier, E. (2011). Bande dessinée : le pari de la matérialité. Communication \& Langages 167.

Fontanille, J. (2005). Paysages, terroirs et icônes du vin. MEI, Le corps, le vin et les images 23, 121-135.

Genette, G. (2004) [1979]. Récit fictionnel, récit factuel, Fiction et diction. Paris : Seuil.

Groensteen, T. (1999). Système de la bande dessinée (Vol. 1). Paris : PUF.

Hennion, A. (2005). Pour une pragmatique du goût. Papiers de Recherche du CSI, 1. Disponible à : http://www.csi.mines-paristech.fr/working-papers/WP/WP_CSI_001.pdf

Hennion, A. (2004). Une sociologie des attachements. D'une sociologie de la culture à une pragmatique de l'amateur. Sociétés 85, 9-23.

Jeanneret, Y. (2008). Penser la trivialité. Volume 1 : La vie triviale des êtres culturels. Coll. Communication, médiation et construits sociaux. Paris : Hermès-Lavoisier.

Kantcheff, C. (2005). La critique sous contraintes. Intervention du 6 octobre 2005 à l'Institut français de la presse (Paris), séminaire La critique impossible ? Disponible à : https://www.politis.fr/blogs/2007/01/la-critique-litteraire-sous-contraintes-142/.

Lambert, E. (2009). Les médiations gustatives, ou l'art de la mise en bouche. Culture \& musées, Scènes et scénographies alimentaires 13, 49-70.

Lambert, E. \& Trouche, D. (2008). L'exposition mise à nu par ses visiteurs, même? Semen, Médiacultures et médiacritiques 26, 81-96. 
Maigret, E. \& Macé, E. (2005). Penser les médiacultures. Nouvelles pratiques et nouvelles approches de la représentation du monde. Paris : Armand Colin / INA.

Pellitteri, M. (2007). Pornography and Synaesthesia in Manga: Multi-sensorial Reception of Eros in Japanese Comics. International Journal of Comic Art 9, 425-440.

Péquignot, B. (2007). Sociologie et médiation culturelle. L'Observatoire 32, 3-7.

Renard, J. (2011). La médiation du manga en France : un lent processus de légitimation. Université de Toulouse : thèse de doctorat en Sciences de l'information et de la communication.

Souchier, E. \& Jeanneret, Y. (1999). L'étiquette des vins : analyse d'un objet ordinaire. Communications et langages 121, 72-85.

Stengel, K. (2017). Les Gestes culinaires : Mise en scène de savoir-faire. Paris : L'Harmattan.

Vanhée, O. (2012). La lecture de manga et ses transformations : enquête sur plusieurs générations de lecteurs en France. Dans E. Maigret \& M. Stefanelli (dir.). La bande dessinée : une médiaculture. Paris : Armand Colin.

\section{(ㅇ) $(\Theta \Theta$}

«Attribution - Pas d'Utilisation Commerciale - Pas de Modification 4.0 International» (CC BY-NC-ND) 